\title{
Controlled attending as a function of melodic and temporal context
}

\author{
MARI RIESS JONES, MARILYN BOLTZ, and GARY KIDD \\ Ohio State University, Columbus, Ohio
}

\begin{abstract}
Melodic and rhythmic context were systematically varied in a pattern recognition task involving pairs (standard-comparison) of nine-tone auditory sequences. The experiment was designed to test the hypothesis that rhythmic context can direct attention toward or away from tones which instantiate higher order melodic rules. Three levels of melodic structure (one, two, no higher order rules) were crossed with four levels of rhythm [isochronous, dactyl (A U U), anapest (U U A), irregular]. Rhythms were designed to shift accent locations on three centrally embedded tones. Listeners were more accurate in detecting violations of higher order melodic rules when the rhythmic context induced accents on tones which instantiated these rules. Effects are discussed in terms of attentional rhythmicity.
\end{abstract}

"Selective attending" traditionally refers to situations in which one listens (or looks) for a particular aspect of stimulation and ignores others. While it has been much researched, it is fair to say that it remains poorly understood. Originally, the phenomenon was studied by Cherry (1953) and Broadbent $(1954,1958)$ using auditory sequences that were simultaneously presented to both ears. These and many subsequent studies verified the importance of physical differences involving, for example, location, pitch, loudness, and so forth, in facilitating a listener's ability to selectively attend to events within one of two cooccurring messages (Egan, Carterette, \& Thwing, 1954; Kahneman, 1973; Moray, 1959; Treisman, 1964). It was also discovered that contextual cues in the form of probabilities and simple grammar constraints of events within messages could be exploited by listeners (Shinar \& Jones, 1973; Treisman, 1960).

It appears that whether one is "set" by physical differences or by probabilistic constraints, performance remains uniformly better on attended messages than on unattended ones (e.g., Moray, Fitter, Ostry, Favreau, \& Nagy, 1976; Shinar \& Jones, 1973; Treisman \& Riley, 1969). Both the fact that performance on the attended task is affected by "unattended" material (Lewis, 1970) and the fact that people can successfully monitor supposedly unattended channels (e.g., Treisman, 1964) contributed to the decline of simple filter models and to the rise of resource capacity models. Thus, according to cur-

The authors are indebted to Robin Wetzel, Shirley Neal, and Robert Ceisler for assistance in data collection. The research was sponsored by Grant BNS-78-06997 awarded to the senior author by the National Science Foundation. Requests for reprints should be sent to Mari Riess Jones, Department of Psychology, Ohio State University, 404-C West 17th Avenue, Columbus, Ohio 43210. rent views, different amounts of attentional resources can be allocated to attended items. Some researchers assume a central capacity resource pool (e.g., Kahneman, 1973), whereas others argue for multiple, task-specific resource pools (e.g., Navon \& Gopher, 1979; Norman \& Bobrow, 1975). Many variations on the resource capacity idea have sprung up. For instance, Massaro claims that restrictions on processing at different stages in an information processing chain operate as capacity limits associated with selective attention (Massaro, 1975, p. 294). Others suggest that, at least with familiar material, there are no real limits to attentional capacity and that attending may be automatic (Posner \& Snyder, 1975; Schneider \& Shiffrin, 1977; Shiffrin \& Schneider, 1977). These capacity limitations emerge largely in tasks involving novel and/or uncertain materials, and here attention must be deliberately controlled.

At first glance, resource capacity models appear plausible. But on closer examination, it is not clear how they parsimoniously apply to attending in many real world environments, environments that consist of highly patterned energy changes over time as in patterns of speech, music, body motions, etc. That is, do we distribute attention to certain parts of those sequences as they unfold in time? And if we do selectively distribute attention, what parts of a pattern capture most attention? What principles determine this allocation? At this level of application, ad hoc principles of attentional allocation appear necessary.

There is an alternative view, one that does not require such ad hoc principles. This view assumes that attending is a rhythmical activity that is naturally controlled, over time, by environmental regularities. Here, pattern invariances (i.e., regularities) encourage the rhythmical attender to differentially focus attention within an unfolding sequence over certain 
anticipated temporal intervals and not over others (Jones, 1976, 1981a).

The present experiment evaluates this rhythmical view of attention. Because it is a view that emphasizes the importance of contextual regularities in guiding attention, it mandates experimental control of serial pattern structure. Therefore, we focus upon the manner in which people distribute attention in time as they listen to musiclike sequences having prescribed serial structure.

There is some recent evidence to suggest that serial patterning in auditory sequences does "set" a listener in ways that enhance recognition of selected embedded elements (Cuddy, Cohen, \& Miller, 1979; Dewar, Cuddy, \& Mewhort, 1977; Meltzer, Martin, Mills, Imhoff, \& Zohar, 1976; Burbridge \& Jones, Note 1). However, it is significant that this research has not manipulated rhythmic context. Yet, if attending is rhythmical, it should be guided by temporal patterning as well. Accordingly, we manipulate the temporal rule structure of sets of musiclike sequences to learn about the effects of rhythm upon listeners' ability to detect deviations from expected melodic rules. In particular, it has been suggested (Jones, $1974,1976,1981 \mathrm{~b})$ that rhythmic context, by inducing different accent locations, may guide attending toward or away from higher order melodic relationships and thus either facilitate or inhibit acquisition of serial rules.

\section{Melodic and Rhythmic Rules}

Here we examine the way contextual regularity encourages people to attend to the "what" and "when" of future events. To do this it is necessary to manipulate contextual regularity, and we outline some of the rules for achieving this end:

(1) Melodic invariants. Sets of rules can specify melodic invariants having different degrees of predictability with respect to frequencies of an auditory sequence. These rules have been described elsewhere (Jones, 1974, 1976, 1978, in press-a, in press-b). Here, we used one such rule group (Figure 1) to manipulate melodic structure in sequences of nine pure tones. The four rules of this set can be defined spatially as flips about axes of a circular $\mathrm{C}$ major scale: identity (I), complement (C), transpose (Tr), and reflection $(\mathrm{Rl})$, which map one scale tone into another.

In the present studies, these rules were applied to an initial three-tone argument to create the longer, nine-tone sequences shown in Table 1. Sequences were generated by the application of one, two, or no rules from the rule set of Figure 1. The resulting patterns contain different degrees of predictability with respect to a common, centrally embedded, three-tone segment.

To illustrate, compare patterns 1,3 , and 5 from Table 1. Each embeds the same central segment, $\mathrm{G}_{4} \mathrm{~A}_{4} \mathrm{~B}_{4}$ (where subscripts refer to octaves). In this segment, a lower order melodic rule relates adjacent tones by " +1 " scale unit. These central tones are related to surrounding tones by either no, one, or two higher order rules, respectively. Thus, for the onerule sequence (pattern 3 ), a repeating transpose rule ensures much predictability:

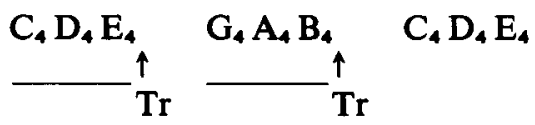

There is less redundancy, but nonetheless noticeable predictability, with the two-rule pattern (pattern 5):

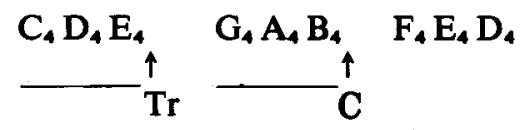

No-rule patterns are control sequences with roughly

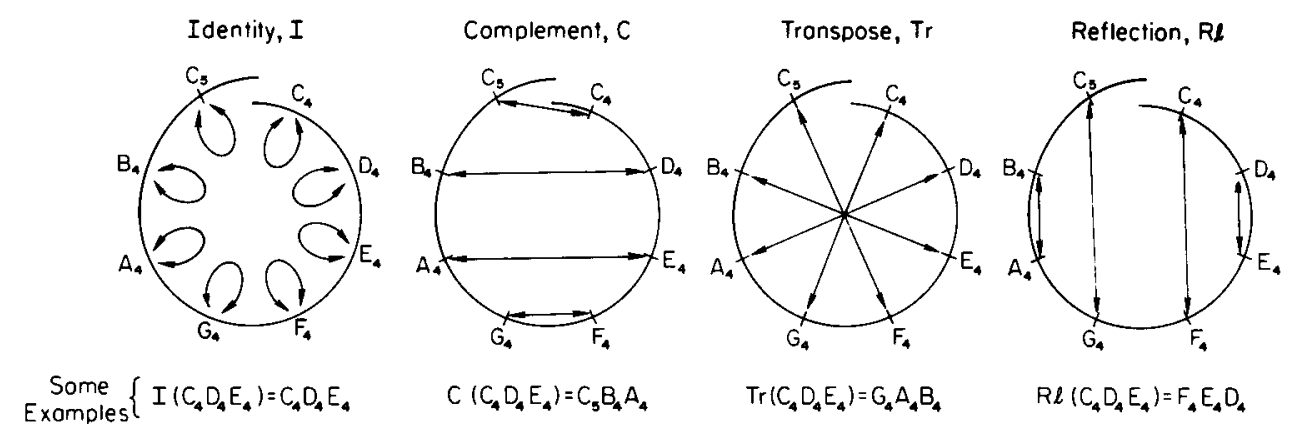

Figure 1. A group of four rules that transform one musical note into another on any diatonic scale shown here as mappings on the $C$ major scale. 
Table 1

Tone Patterns and Generative Rules

\begin{tabular}{|c|c|c|c|c|}
\hline Pattern & & Rules & $\begin{array}{c}\text { Number of } \\
\text { Tones }\end{array}$ & $\begin{array}{c}\text { Number of } \\
\text { Contour Breaks }\end{array}$ \\
\hline \multicolumn{5}{|c|}{ No-Rule Melodies } \\
\hline $\begin{array}{l}1 \\
2\end{array}$ & $\begin{array}{lllll}\mathrm{B}_{3} \mathrm{D}_{4} \mathrm{E}_{4} & \mathrm{G}_{4} \mathrm{~A}_{4} \mathrm{~B}_{4} & \mathrm{~A}_{4} \mathrm{D}_{4} \mathrm{E}_{4} \\
\mathrm{~A}_{3} \mathrm{D}_{4} \mathrm{E}_{4} & \mathrm{~F}_{4} \mathrm{E}_{4} \mathrm{D}_{4} & \mathrm{~A}_{3} \mathrm{E}_{4} \mathrm{~F}_{4}\end{array}$ & $\begin{array}{l}\text { None } \\
\text { None }\end{array}$ & $\begin{array}{l}6 \\
4\end{array}$ & $\begin{array}{l}1 \\
2\end{array}$ \\
\hline \multicolumn{5}{|c|}{ One-Rule Melodies } \\
\hline $\begin{array}{l}3 \\
4\end{array}$ & $\begin{array}{llll}C_{4} D_{4} E_{4} & G_{4} A_{4} B_{4} & C_{4} D_{4} E_{4} \\
C_{4} D_{4} E_{4} & F_{4} E_{4} D_{4} & C_{4} D_{4} E_{4}\end{array}$ & $\begin{array}{l}\text { Transpose } \\
\text { Reflection }\end{array}$ & $\begin{array}{l}6 \\
4\end{array}$ & $\begin{array}{l}2 \\
2\end{array}$ \\
\hline \multicolumn{5}{|c|}{ Two-Rule Melodies } \\
\hline $\begin{array}{l}5 \\
6\end{array}$ & $\begin{array}{lll}\mathrm{C}_{4} \mathrm{D}_{4} \mathrm{E}_{4} & \mathrm{G}_{4} \mathrm{~A}_{4} \mathrm{~B}_{4} & \mathrm{~F}_{4} \mathrm{E}_{4} \mathrm{D}_{4} \\
\mathrm{C}_{4} \mathrm{D}_{4} \mathrm{E}_{4} & \mathrm{~F}_{4} \mathrm{E}_{4} \mathrm{D}_{4} & \mathrm{G}_{4} \mathrm{~A}_{4} \mathrm{~B}_{4}\end{array}$ & $\begin{array}{l}\text { Transpose, Complement } \\
\text { Reflection, Complement }\end{array}$ & $\begin{array}{l}6 \\
7\end{array}$ & $\begin{array}{l}1 \\
2\end{array}$ \\
\hline
\end{tabular}

the same contour (within three-tone arguments) and number of different tones. Thus, pattern 1 is:

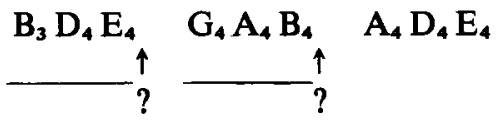

In these sequences, melodic context is evident in two ways. The first involves rule level: lower order rules that relate tones within a three-tone segment (e.g., between $\mathrm{G}_{4} \mathrm{~A}_{4}$ and $\mathrm{B}_{4}$ ) have greater regularity (i.e., frequency) than do higher order rules that relate tones between three-tone segments (e.g., between an initial $C_{4}$ and $G_{4}$ ). Note, then, that a tone occurring at the sixth serial position (SP6) completes a lower order rule, whereas one at the fourth position (SP4) instantiates a higher order rule. The second piece of evidence of melodic context involves higher order rule number: sequences based on one higher order rule have the most redundancy and those with no higher order rule have the least.

(2) Rhythmic invariants. Time rules (i.e., periods) specify "when" different frequencies happen. Rhythm, or relative timing, is expressed through such rules. Essentially, these rules summarize both the lower level time periods of events and interevent pauses and the higher level time periods that overlap these to relate remotely situated accents. Figure 2 illustrates the multileveled nature (i.e., hierarchy) of time rules for four rhythms. The hierarchy that appears above tone durations (labeled numerically by serial position) shows an isochronous rhythm (top row) consisting of 9300 -msec beats, two patterned rhythms (second and third rows) consisting of $12300-\mathrm{msec}$ beats, and an irregular rhythm (bottom row).

Consider first the isochronous (Iso) rhythm. It has all tone durations and all interevent pauses metrically equivalent. Accents derive from many things, including melodic and temporal structure. In this case, there are neither lengthened event durations nor pauses, and, hence, no accenting derives from the pattern's temporal structure. The time rules, $T^{\mathrm{j}}$, shown in Figure 2, refer to time periods between event onsets (i.e., stimulus-onset-asynchrony, SOA). They summarize both time intervals between adjacent (i.e., $\left.T^{1}\right)$ and between remote (e.g., $T^{3}, T^{6}$ ) serial events under the assumption that, in the absence of clear temporal accenting, this nine-tone sequence lends itself to division into three triplets of tones with either dactyl (A U U) or anapest (U U A) accenting for members of successive triplet groups (where A refers to an accented tone and $U$ to an unaccented one).

Two rhythms with coherent temporal patterning are shown below the isochronous sequence. These are $\mathrm{U} U \mathrm{~A}$ and $\mathrm{A} U \mathrm{U}$, respectively. Both include temporally defined accents in the form of metrically lengthened events and pauses (see Figure 2). Each group of three events in these patterns has a total time span of $\mathrm{T}^{4}$, and both contexts, therefore, exhibit
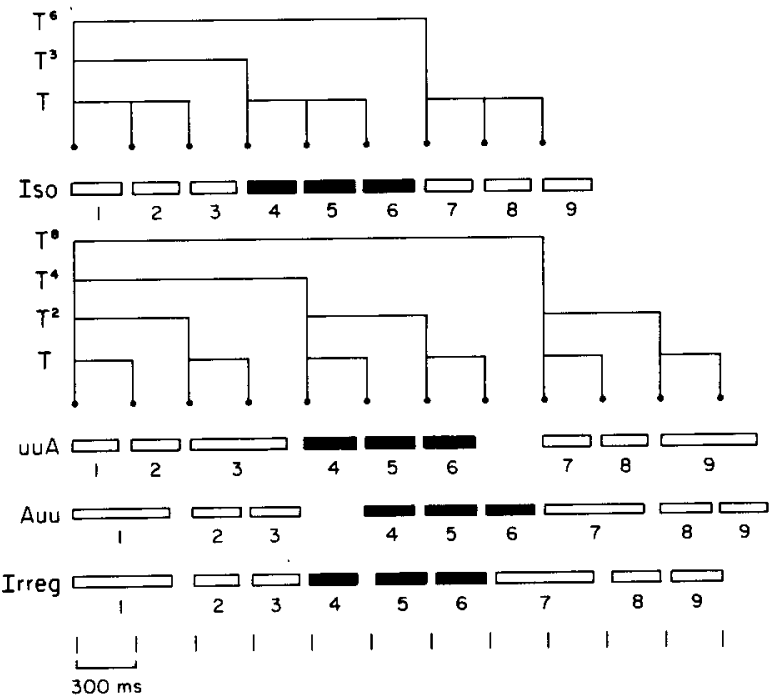

Figure 2. Four rhythmic contexts for a common central segment. The top row shows an isochronous rhythm, the next row, one which induces an accent on the sixth serial position ( $U$ U A), the next, one which induces an accent on the fourth serial position ( $A \mathrm{U} U$ ), and the bottom row, an irregular rhythm. 
temporal hierarchies based upon a multiple of 2 (i.e., $T^{2} / T^{1}=T^{4} / T^{2}=2$ ). Two important points to note about these rhythms are: (a) both embed the same central tone triplets, with the same timing, as found in the isochronous pattern; and (b) the two patterned rhythms differ with respect to temporal locations of accents (the $U$ U A context induces an accent on the sixth tone of the common central segment, whereas the $A \cup U$ induces an accent on the fourth tone of this segment).

The fourth rhythm, shown in Figure 2, is an irregular (Irreg) control sequence that contains some lengthened event durations. Although a slight pause occurs after the fourth event, the time structure of this sequence does not lend itself to a hierarchical summary. This pattern was designed to guide attending away from the fourth serial position.

To summarize, four different temporal contexts induce accents on different serial locations within a common three-tone segment of various melodies. The isochronous and $\mathrm{A} U \mathrm{U}$ rhythms induce an accent on the fourth serial position, while the U U A and the irregular rhythm induce accents at other locations.

\section{Predictions}

In the present experiment, we were interested in exploring the effects of rhythmic context upon melody recognition. We asked listeners to judge whether a transposed comparison pattern was (or was not) identical to a preceding (untransposed) standard sequence. Half the time the pitch of one of the three central tones (at either serial location 4 or 6 ) was slightly altered. Different groups of listeners judged various melodic sequences in, respectively, four different rhythmic contexts that corresponded to those of Figure 2.

If attention is rhythmically guided, then listeners receiving the isochronous and $A U U$ contexts, which prepare one, in advance, to attend to the fourth serial location, should be better (at recognizing melodic differences) at these points than would listeners in other conditions. Since rule violations at the fourth serial location involve higher order rules, this prediction reflects the hypothesis that rhythmic accenting can facilitate higher order rule learning. Similarly, listeners receiving rhythms designed to accent the sixth serial position, namely, those hearing isochronous and $U \mathbf{U} A$, should perform better than other groups in recognizing melodic differences (involving lower order rules) which occur at these locations.

\section{METHOD}

\section{Subjects}

Forty-eight students from an introductory psychology class at Ohio State University volunteered to serve in this experiment in return for course credit. They were randomly assigned $(n=6)$ to each of the rhythm and counterbalance-order conditions.

\section{Design}

The design was a $4 \times 3 \times 3 \times 2$ mixed factorial. Four levels of rhythm (Iso, U U A, A U U, and Irreg) were crossed with three levels of melodic context $(0,1,2$ rules). Three levels of serial position (same, different at SP4, and different at SP6) were used. Trials were presented in two random counterbalance orders (I, II). Rhythm and counterbalance order were the only between-subject variables.

\section{Apparatus}

All patterns were composed of pure tones generated by a Wavetek Model 159 waveform generator controlled by a Cromemco A-2 microcomputer. A given tone sequence was recorded on a Nakimichi 550 cassette recorder. Sets of prerecorded sequences were presented through an Epicure Model 5 loudspeaker set at a comfortable listening level, which remained constant throughout the experimental sessions.

\section{Stimulus Materials}

Within each rhythm condition, a total of $\mathbf{4 0}$ nine-tone pattern pairs (standard-comparison) were constructed. In all cases, the comparison sequence was untransposed relative to the standard, although it could differ from the standard with respect to either the fourth or sixth note. Half of the comparisons did not differ (i.e., "sames"), and half included deviant tones (i.e., "differents").

\section{Pattern Construction}

Frequency patterns of tones were generated by systematic application of melodic rules (see Table 1) to one of two three-tone arguments. The resulting patterns are listed in musical notation in Table 1; frequencies follow $A_{4}=440 \mathrm{~Hz}$ standardization. Generating rules were applied to the $\mathrm{C}$ major scale under the assumption of equal scale unit intervals.

For each standard frequency pattern, three comparison patterns were possible, one which was identical to the standard pattern and two which included a deviant tone. The deviant tones in the comparison patterns occurred in either the fourth or sixth serial position of the nine-tone sequence. To achieve a completely balanced design, an equal number of identical comparisons were always associated with a given standard (i.e., since there were two deviant comparison patterns for each standard, there were also two identical comparison patterns for each standard).

Construction of the deviant comparison melodies conformed to the following constraints: (1) Deviant tone sequences were not permitted to break the contour established by the standard sequence. (2) No deviant tone was repeated in succession in the ninetone sequence. (3) Deviant tones from the $\mathrm{C}$ major triad (C, E, G) were avoided due to their high musical familiarity. (4) All deviant tones were of the $C$ major scale. (5) Changed tones could not create a repeating tone. (6) Deviations were between 1 and 3 scale units.

Melodic rule conditions. Across all three rule conditions $(0,1$, 2 rules), the same central three-tone segment appeared in different contours. The resulting six experimental patterns consisted of two pattern instances for each of the three rule levels. As shown in Table 1, common central segments were $G_{4} A_{4} B_{4}$ and $F_{4} E_{4} D_{4}$.

One-rule condition. In the one-rule condition, one rule was applied to the initial three-tone argument to yield the central three-tone segment and then was applied once more to complete the nine-tone pattern. Two standard melodies were created using the reflection and transpose rules, respectively.

Two-rule condition. The two-rule condition involved successive applications of two different rules to the initial three-tone segment. One standard melody was created by successive applications 
of the transpose and complement rules and another by application of the reflection and complement rules. This resulted in patterns that differed from one-rule sequences only with respect to the final three tones. These patterns also necessarily differed from onerule sequences in terms of number of different tones engaged and, in some cases, in contour.

No-rule patterns. Six standard no-rule patterns that embedded the same three-tone segment as rule-governed sequences were constructed. Control sequences may be constructed in a variety of ways, depending upon their function (cf. Jones, 1981b). In the present studies, no-rule patterns were devised to preserve the following commonalities with other rule-governed sequences: same central three-tone segment; same total number of different tones; as many chromatically similar tones as possible; similar contour within each of the three three-tone melodic chunks (i.e., no patterns contained more than two contour breaks).

Temporal rule conditions. Four different rhythmic forms corresponding to those illustrated in Figure 2 were presented, respectively, to different groups of subjects. These rhythmic conditions were:

Isochronous rhythm condition (Iso). All SOA's were $300 \mathrm{msec}$ and were, in turn, composed of tonal durations of $250 \mathrm{msec}$ and silent intertone intervals of $50 \mathrm{msec}$.

Patterned rhythm, anapest ( $U U A$ ). All event durations and intertone intervals remained, respectively, $250 \mathrm{msec}$ and $50 \mathrm{msec}$, with the following exceptions: events at the third and ninth (i.e., final) serial locations lasted $500 \mathrm{msec}$; intertone intervals between the third and fourth events were $100 \mathrm{msec}$; and intertone intervals between the sixth and seventh serial events were $350 \mathrm{msec}$. Both lengthened events and pauses were metrically crafted to jointly produce strong temporal accents on events occurring at serial positions 3 (SP3) and 9 (SP9), and thereby induce accenting on the event occurring at serial position 6 (SP6).

Patterned rhythm, dactyl ( $A U U$ ). All event durations and intertone intervals were, respectively, $250 \mathrm{msec}$ and $50 \mathrm{msec}$, with the following exceptions: on and off times were $500 \mathrm{msec}$ and $100 \mathrm{msec}$, respectively, for serial events 1 (SP1) and 7 (SP7); the intertone interval between events at serial locations 3 and 4 was lengthened to $350 \mathrm{msec}$. In this rhythm, the metrics of durations and pauses were designed to induce accenting on the fourth serial event (SP4).

Irregular. The irregular rhythm was identical to the A U U, with two exceptions: the intertone interval between events 3 and 4 was $50 \mathrm{msec}$ (not $350 \mathrm{msec}$ ); the intertone interval between events 4 and 5 was lengthened to $100 \mathrm{msec}$ (from $50 \mathrm{msec}$ ). These time changes were designed to invalidate the accenting of SP4 established by durations and pauses of $A \mathrm{U} U$.

Experimental and dummy patterns. Within each block of 40 trials in a given rhythm condition, 10 different pattern types occurred. Six were experimental pattern types ( 2 pattern instances/ rule level), and 4 were dummy patterns. Each of these 10 pattern types occurred 4 times/block: twice in "same" pairs; once as "different" SP4; once as "different" SP6. Two blocks of forty trials were used.

Dummy pattern pairs were designed for two functions: to discourage subjects from developing a strategy of attending only to serial locations 4 and 6; to discourage subjects from developing strategies specific to rule level manipulations. In dummy patterns, a variety of rule and no-rule forms could occur and the changed notes could happen at any serial location between 2 and 8 , inclusive.

\section{Procedure}

Listeners were informed, via recorded instructions, to judge the melodic equivalence of standard and comparison nine-tone sequences. On each trial, they indicated whether or not the comparison pattern was the same as or different from the standard pattern. In making their judgments, subjects were also asked to indicate their confidence in their ratings. Alternatives were: "same," " sure same," "different," "sure different."
Each trial was preceded by a warning tone $(5,000 \mathrm{~Hz})$ that lasted $1 \mathrm{sec}$. Two seconds intervened between standard and comparison sequences on each trial, and, following the comparison, a 10-sec silent period occurred before the next warning tone. During this 10 -sec period, subjects judged the preceding pattern pair.

\section{RESULTS AND DISCUSSION}

Mean proportion correct (PC) averaged over counterbalance order in each of the four rhythm condititions are in Table 2. Of major interest are the effects of position (same, SP4, SP6) and rhythm. Table 3 presents melodic context effects.

Considering serial position first, subjects found it more difficult to recognize violations of higher order rules (SP4) than to recognize either violations of lower order rules (SP6) or no violations (same) $[\mathrm{F}(2,120)$ $=53.45, \mathrm{p}<.001, \mathrm{MSe}=.043]$. On the average, $\mathrm{PC}$ was .66 at the higher order rule transition point identified with SP4, and it was .865 and .835 for same and different, respectively, at SP6. These differences as a function of melodic rule level were present in some degree in all types of melodic sequences (Table 2) and in all rhythmic conditions (Table 3).

An interaction of melodic context with position does occur $[\mathrm{F}(4,240)=4.35, \mathrm{p}<.005, \mathrm{MSe}=.032]$, but it does not reflect a significant modulation of performance accuracy in detecting lower vs. higher level rule violations. Instead, it appears that people were exceptionally good at recognizing the unchanged onerule patterns. Table 3 indicates that "same" responses were over $10 \%$ more accurate on the one-rule melodies than on the two-rule and no-rule sequences. Since improved performance on the one-rule melodies is not at the expense of significantly lowered accuracy

Table 2

$P C$ as a Function of Melodic Context

\begin{tabular}{lcccc}
\hline & \multicolumn{4}{c}{ Serial Position } \\
\cline { 2 - 4 } Melodic & & \multicolumn{2}{c}{ Different } \\
\cline { 2 - 4 } Context & Same & SP4 & SP6 & Mean \\
\hline No Rule & .809 & .676 & .824 & .760 \\
One Rulc & .947 & .645 & .836 & .809 \\
Two Rules & .842 & .668 & .844 & .788 \\
Mean & .866 & .663 & .835 & .788 \\
\hline
\end{tabular}

Table 3

$P C$ as a Function of Rhy thmic Context

\begin{tabular}{lcccc}
\hline & \multicolumn{4}{c}{ Serial Position } \\
\cline { 2 - 4 } \multicolumn{1}{c}{ Rhythm } & \multicolumn{4}{c}{ Different } \\
\cline { 2 - 4 } & Same & SP4 & SP6 & Mean \\
\hline Isochronous & .891 & .729 & .828 & .816 \\
U U A & .859 & .583 & .880 & .774 \\
A U U & .844 & .740 & .807 & .797 \\
Irregular & .870 & .599 & .823 & .764 \\
Mean & .866 & .663 & .835 & .788 \\
\hline
\end{tabular}


at SP4 or SP6, it appears that the greater redundancy of one-rule melodies translates largely into a clearer concept of the pattern itself.

The most important finding is that rhythmic context modulates effects of melodic rule level. Although no main effect of rhythm was obtained, a significant interaction of rhythm with position is shown in Table $3[F(6,120)=3.72, p<.0025, \mathrm{MSe}=.043]$. The effect is in the expected direction: $U$ U A and irregular rhythms produce poorer performance with rule violations at SP4 than do A U U and isochronous rhythms. Similarly, for the sixth serial position, performance was best for the condition that encouraged attending to this event location, namely, U U A. Post hoc analysis using a components analysis of variance indicated that reliable effects of rhythmic differences occurred with recognition of pitch changes on SP4. At this serial location, rhythmic context produced a significant overall effect $[F(3,60)=4.39$, $\mathrm{p}<.007]$. Other contrasts showed that the A U U rhythm produced superior performance when compared with both the U U A $[F(1,30)=5.09, p<.005]$ and. with the irregular rhythms $[F(1,30)=11.39$, $\mathrm{p}<.0025$ ], but not when compared with the isochronous rhythm $(p>.05)$. At the sixth serial position, performance was generally high (PC was always above .80 ), and although the rhythm designed to accent this location, namely, $U$ U A, did produce best performance $(\mathrm{PC}=.88)$ and the one designed to accent SP4, namely, A U U, produced poorest performance $(P C=.807)$, these differences were not statistically significant.

Finally, counterbalance order had some significant effects in this second study, although these effects do not qualify the major findings. People receiving one counterbalance (I) order were more accurate than those receiving the other (II) $[\mathrm{F}(1,56)=8.96, \mathrm{p}<.005$, $\mathrm{MSe}=.125]$. In part, this results from an interaction of position with melodic rule condition an counterbalance order: listeners in counterbalance order 1 were relatively more accurate on SP6 with no-rule sequences than were listeners in order II $[F(4,224)$ $=2.44, \mathrm{p}<.05, \mathrm{MSe}=.032]$. Since these effects were not affected by rhythm, they do not qualify the major conclusions of this experiment. However, they do reinforce the need to understand carry-over effects that stem from patterning over trials within larger experimental contexts (cf. Jones, Kidd, \& Wetzel, 1981).

In summary, these results lead to several conclusions: recognition of pitch changes is more difficult at higher order rule transitions than at lower order ones; unchanged melodies are recognized best when they are very simple; and rhythmic context can, by induced accenting, modulate position effects such that when higher order rule transitions are accented, correct detection of rule violations increases and when higher order rule transitions are not accented, correct detection of rule violations decreases.

\section{GENERAL DISCUSSION}

It appears that both melodic and rhythmic context affect recognition of embedded tones. People are better at recognizing tones that violate predictable higher level rules. And rhythm modulates these melodic effects. That is, temporal predictability enhances detection of higher order melodic structure.

There are specific effects of melodic regularity that are evident primarily in two findings. First, listeners are generally more accurate at detecting violations of lower order rule transition (SP6) than they are with higher order rule transitions (SP4). And second, transposed one-rule melodies are especially easy to identify in their unchanged form.

These findings are particularly interesting for the converging evidence they provide with results from serial pattern anticipation research that has involved learning and memory of nonauditory time patterns (e.g., Jones, 1974; Jones \& Zamostny, 1975; Restle \& Brown, 1970). Traditionally, serial pattern learning studies have shown that people make more anticipation errors in learning to predict those events associated with higher order rule instantiations. The present data reveal something else. They show that people also make more errors in recognizing violations of higher order rules. This highlights a useful distinction. That is, in view of the present findings, recognition of differences appears to depend, in part, upon one's ability to correctly anticipate what should occur at a particular time. In addition, the finding that intact instances of the more redundant, one-rule sequences are easier to recognize is consistent with recent work in music recognition that emphasizes listeners' use of simple prototypes (Cuddy, Cohen, \& Mewhort, 1981).

The most important finding emerging from the present research has to do with the fact that rhythmic context modulates the recognition accuracy of higher order rule violations. Although Dewar et al. (1977) speculated that the accent/rhythm hierarchy underlying their melodies might account for certain serial position effects they observed in a tonal recognition task, no other research has illustrated these effects. The fact that rhythmic context may guide attending either toward or away from particular times supports the original hypothesis that attending is rhythmical. The A U U rhythm along with the isochronous rhythm facilitated the detection of higher order rule violations. In comparing performance in the two rhythmic conditions that possess coherent temporal accenting, namely, $U$ U A and $A U U$, it is apparent that when temporal accents induce an accent on the sixth serial position, as in $U$ U A, then recognition is relatively poor for changes that happen on the fourth serial location. Both the U U A and irregular rhythmic context were designed to ensure that induced accents would not occur at SP4, the higher order rule transition. In both conditions, performance on SP4 was, 
indeed, relatively poor. The preferred explanation is that attention is being directed by the structure of these contexts so as to be maximal on SP6; hence, less attention is allocated to SP4.

There is some evidence that rhythmic context facilitates detection of lower level rule violations, but this is less clear. Although performance on the SP6 event was better in those rhythmic conditions designed to induce accenting on these locations, these differences were not statistically significant. The most obvious explanation of this finding involves a ceiling effect on performance: for all groups, recognition performance of lower order rule violations on SP6 averaged over $80 \%$ correct.

These findings have both theoretical and practical significance. Theoretically, they suggest how higher level time rules (between accents) can facilitate detection of higher level melodic rules. The time structure of a sequence can encourage selective attending to tones which document higher order rules. Thus, the melodic figures that emerge for a listener and that can emphasize these rules depend upon selective attending in time. Neisser and Becklen (1975), in illustrating the importance of the structured flow of visual patterns, have provided related examples of selective attending in vision, in which spatial relationships also play a controlling role. However, with auditory patterns, selective attention to accented tones within a single coherent melodic line involves different levels of attending (i.e., levels of time structure within a pattern), and thereby it emphasizes the hierarchical nature of dynamic attending (Jones, 1976; Martin, 1972).

Practically speaking, these findings dovetail nicely with well-known tricks of musical composition that often involve elements of well-timed surprise. The artful composer who wishes to ensure maximal listener appreciation of a clever musical embellishment (i.e., rule violation) places this "surprise" at an accented time location (Jones, in press-b). Rhythmic accenting may also simply emphasize the onsets of phrases and so suggest a particular musical phrase structure. Or it may be used to strengthen or weaken tonality by emphasizing certain pitch relationships. These are only a few of the ways in which rhythmic structure is typically manipulated to guide attention.

It is difficult to square the pattern of these findings with traditional approaches to attending. Most resource capacity models simply fail to consider attending to complex dynamic patterns. And those that do, place a heavy explanatory burden upon backward masking. In Massaro's approach (Idson \& Massaro, 1976; Massaro, 1975), for example, time constraints on attentional resources can reduce processing effectiveness at the tone recognition stage by increasing backward masking. Instead of preparing a listener in advance, here time relations affect encoding after a tone has occurred. Thus, an SOA between a target and a subsequent tone (potential mask) that is much below $200 \mathrm{msec}$ will hamper target pitch recognition. ${ }^{1}$ This model cannot explain these rhythmic context effects because the SOA's involved exceed $250 \mathrm{msec}$. His masking interpretation emphasizes constraints of brief time periods within either the standard or comparison sequences. Consequently, there is no reason to expect systematic differences due to temporal context on detection of changed events at serial positions four and six. The lawful effects due to temporal context observed in these studies indicate that the impact of rhythm on attending in these models has been ignored.

An alternative explanation might hold that some rhythm contexts, because they include pauses, will "parse" the melody in optimal or nonoptimal ways. This line has been taken by Deutsch (1980). Unfortunately, the term "parse" is imprecise (see Jones, $1981 \mathrm{~b}$, for a discussion). If we assume that any lengthened time interval can "parse" a sequence, then the embedded central segment will "stand out" equally well in both patterned rhythms (i.e., $U$ U A and A U U), thus incorrectly predicting no differences between the two patterned rhythms. Even if "parsing" occurs only with increased intertone silent intervals, it is not clear what qualifies as a "parse": while both $U U A$ and $A U U$ have pauses prior to the fourth event, the pause of $A U$ U is longer. And other aspects of the data are unexplained. For example, performance on the temporally predictable isochronous sequence, which contains no pauses, is often better than in those with pauses. Furthermore, this explanation cannot account for the uniformly good performance on SP6 even in the A U U and the irregular rhythms, in which no pause follows this position. It is probably more useful to consider the metric implications of a pause within the context of a larger rhythmic hierarchy than to argue that pauses merely segment a pattern. Emphasis upon the metrics of pauses and durations reconciles this work with that of others that emphasize interval and ratio properties of time structure (e.g., Handel \& Oshinsky, 1981; Jones, 1976; Martin, 1972; Povel, 1981). A simple parsing account ignores details of accenting and so cannot explain differential accuracy at SP4 and SP6 as a function of time metrics. Yet, it is accenting, defined in part by a pause, which actually groups tones. The present data suggest that it is more useful to consider the metric hierarchy of a rhythmic context and the way in which it encourages specific focusing of attention.

\section{REFERENCE NOTE}

1. Burbridge, S., \& Jones, M. R. The function of rule-governed serial contexts in recognition of embedded tones. Manuscript submitted for publication, 1982. 


\section{REFERENCES}

Broadeent, D. E. The role of auditory localization and attention in memory span. Journal of Experimental Psychology, 1954, 47, 191-196.

Broadbent, D. E. Perception and communication. New York: Pergamon Press, 1958.

Cherry, E. C. Some experiments on the recognition of speech, with one and with two ears. Journal of the Acoustical Society of America, 1953, 25, 975-979.

Cuddy, L. L., Cohen, A. J., \& Mewhort, D. J. K. Perception of the structure in short melodic sequences. Journal of Experimental Psychology: Human Perception and Performance, 1981, 7, 869-883.

Cuddy, L., Cohen, A., \& Miller, J. Melody recognition: The experimental application of musical rules. Canadian Journal of Psychology, 1979, 33, 148-157.

DEUTSCH, D. The processing of structured and unstructured tonal sequences. Perception \& Psychophysics, 1980, 28, 381-389.

Dewar, K. M., Cuddy, L. L., \& Mewhort, D. J. K. Recognition memory for single tones with and without context. Journal of Experimental Psychology: Human Learning and Memory, 1977, 3, 60-67.

Egan, J. P., Carterette, E. C., \& Thwing, E. J. Some factors affecting multichannel listening. Journal of the Acoustical Society of America, 1954, 26, 774-782.

Handel, S., \& Oshinsky, J. S. The meter of syncopated auditory polyrhythms. Perception \& Psychophysics, 1981, 30, 1.9.

Ioson, W. L., \& Massaro, D. W. Cross-octave masking of single tones and musical sequences: The effects of structure on auditory recognition. Perception \& Psychophysics, 1976, 19, 155-175.

Idson, W. L., \& Massaro, D. W. A bidimensional model of pitch in the recognition of melodies. Perception \& Psychophysics, 1978, 24, 551-565.

Jones, M. R. Cognitive representations of serial patterns. In B. Kantowitz (Ed.), Human information processing: Tutorials in performance cognition. Potomac, Md: Erlbaum, 1974.

JoNEs, M. R. Time, our lost dimension: Toward a new theory of perception, attention, and memory. Psychological Review, 1976, 83, 323-355.

Jones, M. R. Auditory patterns: The perceiving organism. In E. C. Carterette \& M. P. Friedman (Eds.), Handbook of perception (Vol. 8). New York: Academic Press, 1978.

Jones, M. R. Only time can tell: On the topology of mental space and time. Critical Inquiry, 1981, 7, 557-576. (a)

JONES, M. R. A tutorial on some issues and methods in serial pattern research. Perception \& Psychophysics, 1981, 30, 492-504. (b)

JONES, M. R. Music as a stimulus for psychological motion: Part I. Some determinants of expectancies. Psychomusicology, in press. (a)

JoNEs, M. R. Music as a stimulus for psychological motion: Part II. An expectancy model. Psychomusicology, in press. (b)

Jones, M. R., KIDD, G., \& WETzel, R. Evidence for rhythmic attention. Journal of Experimental Psychology: Human Perception and Performance, 1981, 7, 1059-1073.

Jones, M. R., \& Zamostny, K. P. Memory and rule structure in the prediction of serial patterns. Journal of Experimental Psychology: Human Learning and Memory, 1975, 1, 295-306.

Kahneman, D. Attention and effort. Englewood Cliffs, N.J: Prentice Hall, 1973.

LEwis, J. L. Semantic processing of unattended messages using dichotic listening. Journal of Experimental Psychology, 1970, 85, 225-228.

Martin, J. Rhythmic (hierarchical) vs. serial structure in speech and other behavior. Psychological Review, 1972, 79, 487-509.
Massaro, D. W. Experimental psychology and information processing. Chicago: David MacNally, 1975.

Meltzer, R. H., Martin, J. G., Mills, C. B., Imhoff, D. L., \& Zohar, D. Reaction time to temporally-displaced phoneme targets in continuous speech. Journal of Experimental Psychology: Human Perception and Performance, 1976, 2, 277-290.

Moray, N. Attention in dichotic listening: Affective cues and the influence of instructions. Quarterly Journal of Experimental Psychology, 1959, 11, 56-60.

Moray, N., FitTer, M., Ostry, D., Favreau, D., \& Nagy, V. Attention to pure tones. Quarterly Journal of Experimental Psychology, 1976, 28, 271-283.

Navon, D., \& Gopher, D. On the economy of the humanprocessing system. Psychological Review, 1979, 86, 214-255.

Neisser, U., \& Becklen, R. Selective looking: Attending to visually specified events. Cognitive Psychology, 1975, 7, 480-494.

Norman, D. A., \& Boвnow, D. G. On data-limited and resource limited processes. Cognitive Psychology, 1975, 7, 44-64.

Posner, M. I., \& SNYder, C. R. R. Attention and cognitive control. In R. L. Solso (Ed.), Information processing and cognition. Hillsdale, N.J: Erlbaum, 1975.

Povel, D. J. Internal representation of simple temporal patterns. Journal of Experimental Psychology: Human Perception and Performance, 1981, 7, 3-18.

Restle, F., \& Brown, E. R. Organization of serial pattern learning. In G. H. Bower (Ed.), The psychology of learning and motivation: Advances in research and theory (Vol. 4). New York: Academic Press, 1970.

Schneider, W., \& Shiffrin, R. Controlled and automatic human information processing. 1. Detection, search and attention. Psychological Review, 1977, 84, 1-66.

Shirfrin, R. M., \& Schneider, W. Controlled and automatic human information processing. II. Perceptual learning, automatic attending, and a general learning. Psychological Review, $1977,84,127-190$.

ShinaR, D., \& Jones, M. R. Effects of set-inducing instructions on recall from dichotic inputs. Journal of Experimental Psychology, 1973, 98, 239-245.

Treisman, A. M. Contextual cues in selective listening. Quarterly Journal of Experimental Psychology, 1960, 12, 242-248.

Treisman, A. M. The effect of irrelevant material on the efficiency of selective listening. American Journal of Psychology, 1964,77, 533-546.

Treisman, A., \& Riley, J. Is selective attention selective perception or selective response? A further test. Journal of Experimental Psychology, 1969, 79, 27-34.

\section{NOTE}

1. The masking argument as developed by Idson and Massaro (1976) would apply to successive tones within, respectively, standard and comparison sequences. Effects of masking on the recognition of transposed comparisons should, however, also occur, given the arguments of these workers. Indeed, they have maintained elsewhere (Idson \& Massaro, 1978) that chromatic similarities between tones an octave apart are powerful equivalences. Thus, the fact that the comparison sequences are transposed up one octave in the present experiments should not qualify these predictions.

(Manuscript received February 5, 1982; revision accepted for publication May 17,1982 .) 